\title{
Searching satellites of asteroid Itokawa by imaging observation with Hayabusa spacecraft
}

\author{
Tetsuharu Fuse $^{1}$, Fumi Yoshida ${ }^{2}$, David Tholen $^{3}$, Masateru Ishiguro $^{4}$, and Jun Saito ${ }^{5}$ \\ ${ }^{1}$ Subaru Telescope, National Astronomical Observatory of Japan, Hilo, HI 96720, USA \\ ${ }^{2}$ National Astronomical Observatory of Japan, Mitaka, Tokyo 181-8588, Japan \\ ${ }^{3}$ Institute for Astronomy, University of Hawaii, Honolulu, HI 96822, USA \\ ${ }^{4}$ Department of Astronomy, Seoul National University, Kwanak-gu, Seoul 151-747, Korea \\ ${ }^{5}$ Satellite Business Division, PASCO Corporation, Meguro, Tokyo 153-0043, Japan
}

(Received November 28, 2006; Revised January 25, 2008; Accepted January 29, 2008; Online published February 12, 2008)

\begin{abstract}
We carried out a search for satellites around Itokawa, the target asteroid of the Japanese Hayabusa mission. An imaging instrument, AMICA, on the spacecraft was used to take four images of Itokawa and its vicinity on September 1, 2005. The distance of the spacecraft from Itokawa was approximately $1,700 \mathrm{~km}$, somewhat longer than the original plan $(1,000 \mathrm{~km})$ due to the mission schedule. The field-of-view of AMICA corresponded to $170 \mathrm{~km} \times 170 \mathrm{~km}$ around Itokawa. Since the Hill sphere of Itokawa was estimated to be $33 \mathrm{~km}$ at the time of the observations, the images completely covered the detectable area of satellites. However, we cannot find the motion of Itokawa satellites between the four images, because the observation period was set to $2 \mathrm{hr}$ due to the mission schedule and the region where satellites would be observable was only within $8 \mathrm{~km}$ of Itokawa, which is inside the extensive glare of Itokawa on the images. In addition, high-energy protons produced by a huge solar flare impacted the spacecraft during the observations, and hence the four images suffered from many random spots. Comparing the positions of the spots with catalogued stars, we managed to identify stellar images. No evidence of satellites was found. Additionally, we evaluated the 1-m detection limit of the images from the limiting magnitude of 9.5. The absence of satellites of Itokawa is consistent with past optical and radar observations as well as other results by the Hayabusa spacecraft.
\end{abstract}

Key words: Asteroid, satellite, space mission.

\section{Introduction}

The existence of satellites around asteroids or asteroidal binary systems was suggested by lightcurve observation over 100 years ago (Merline et al., 2002). Double craters on planets are thought to be evidence for the existence of binary systems (Melosh and Stansberry, 1991). Previous studies have indicated that the formation of asteroidal satellites and binary systems could result from planetary tidal forces, fragmentation from catastrophic collisions, ejection from impact craters, or rotational fission (Pravec et al., 2006; Merline et al., 2002). Investigations into asteroidal satellites and binary systems are expected to help characterize the physical properties of asteroids and provide clues to the history of the Solar System's evolution.

Asteroid 243 Ida and its companion Dactyl were the first asteroidal satellite system to be discovered when the Galileo spacecraft flew by in 1993 (Chapman et al., 1995; Belton et al., 1996). Since radar and adaptive optics observation with large telescopes have recently become available, tens of asteroids with a nearby satellite (and two satellites for 87 Sylvia) have been found. The mass of an asteroid, one of the most important physical parameters, can generally be determined by Kepler's law using the orbital period and

Copyright (c) The Society of Geomagnetism and Earth, Planetary and Space Sciences (SGEPSS); The Seismological Society of Japan; The Volcanological Society of Japan; The Geodetic Society of Japan; The Japanese Society for Planetary Sciences; TERRAPUB. orbital radius of the satellite within the binary system.

Near-Earth asteroid 25143 Itokawa (1998 SF36), classified as an S-type, was discovered on September 26, 1998 by the LINEAR project (Williams, 1998). Because it has high accessibility for spacecraft due to its orbital characteristics (Table 1), Itokawa was selected as the target object for the Japanese space probe Hayabusa (Fujiwara et al., 2006). Although the spacecraft was mainly designed for space engineering development, it also carries scientific instruments to conduct observations. Among the instruments, the Asteroid Multiband Imaging Camera (AMICA) serves a dual role of navigation camera (at that time, called ONC-T) and scientific imaging camera. We anticipated that satellites could be discovered by imaging the vicinity around Itokawa with AMICA during the Hayabusa approach phase. Since the detection limit of our observations was expected to be in the meter-scale size range, there was a high probability of discovering Itokawa's satellites, if present.

Before the Hayabusa spacecraft arrived at Itokawa, there had been many earth-based observations of Itokawa. Some of the most notable were the radar observations in 2001 that revealed the flattened ellipsoidal shape of Itokawa and confirmed its size of $548 \mathrm{~m} \times 312 \mathrm{~m} \times 276 \mathrm{~m}$ (Ostro et al., 2004). Further radar observations suggested a total volume of $2.41 \times 10^{-2} \mathrm{~km}^{3}$ (Scheeres et al., 2004). The density of an S-type asteroid is generally estimated to be approximately 2.6 to $2.7 \mathrm{~g} / \mathrm{cm}^{3}$ (Britt et al., 2002); however, 
Table 1. The orbital elements of Itokawa at Epoch 2006/9/22.0 TDB from JPL Solar System Dynamics at http://ssd.jpl.nasa.gov/. a is the semi-major axis, $e$ is the eccentricity, $\omega$ is the argument of perihelion, $\Omega$ is the longitude of the ascending node, $i$ is the inclination, $M$ is the mean anomaly, and $P$ is the orbital period. $r$ is the heliocentric distance on $2005 / 9 / 1.0$ in UTC calculated by JPL HORIZONS system at the same web site.

\begin{tabular}{cccccccc}
\hline$a(\mathrm{AU})$ & $e$ & $\omega(\mathrm{deg})$ & $\Omega(\mathrm{deg})$ & $i(\mathrm{deg})$ & $M(\mathrm{deg})$ & $P($ day $)$ & $r(\mathrm{AU})$ \\
\hline 1.3239 & 0.28008 & 162.77 & 69.095 & 1.6222 & 193.31 & 556.41 & 1.2073 \\
\hline
\end{tabular}

assuming a density of $2.5 \mathrm{~g} / \mathrm{cm}^{3}$, we derived a mass of $6.01 \times 10^{10} \mathrm{~kg}$ for Itokawa from the volume estimated by Scheeres et al. (2004). We found that if the existence of satellites were confirmed, it would help to evaluate the mass of Itokawa determined using other scientific instruments on the Hayabusa spacecraft.

The findings from previous radar studies were important to this study because the radar beam was about $1,000 \mathrm{~km}$ wide at Itokawa's distance (Ostro et al., 2004), almost equal to our search range. The radar spatial resolution is a few decameters and a satellite's motion should not be much faster than meters per second. The detection limit of our observation is expected to be a meter-scale size, and therefore high probability of the discovery of satellites by AMICA imaging.

\section{Observation}

A satellite around an asteroid has gravitational influence from not only the asteroid but also the sun; however, the effect of solar gravity becomes smaller than the asteroid's gravity inside its Hill sphere. If we assume the orbital eccentricity of Itokawa is negligible (i.e. a circular orbit; cf. Table 1), a satellite should be inside the Itokawa's Hill sphere $R_{H}$ (e.g. Hamilton and Burns, 1991):

$$
R_{H}=\left(\frac{m}{3 M}\right)^{1 / 3} r
$$

where $m$ and $M$ are the mass of Itokawa and the sun, respectively, and $r$ is the heliocentric distance of Itokawa. We first used the mass of Itokawa by Scheeres et al. (2004) to estimate the Hill sphere using Eq. (1) and determined the observing plan. The accurate mass of $(3.51 \pm 0.105) \times 10^{10} \mathrm{~kg}$ is now available from the Hayabusa observations (Fujiwara et al., 2006). This is approximately half the mass estimated by Scheeres et al. (2004).

During approaching and hovering phases between August and December of 2005, we obtained remarkable physical information on Itokawa from the spacecraft observations. Due to the research objective constraints, we could only search for Itokawa's satellites during the spacecraft's approaching phase at the end of August or beginning of September of 2005. This viewing window was when the distance between the spacecraft and Itokawa was expected to be equal to, or less than, 1,000 km. We first evaluated the search area, ensuring it was wider than the Hill sphere of Itokawa, by taking into account the specifications of AMICA and Itokawa's heliocentric distance.

Figure 1 shows the variation of the Hill sphere of Itokawa, assuming a mass of $3.51 \times 10^{10} \mathrm{~kg}$, between $\mathrm{Au}-$ gust and December of 2005. This term corresponds to the approaching and departing phases of the spacecraft. The Hill sphere during the period varies roughly from $36 \mathrm{~km}$ to

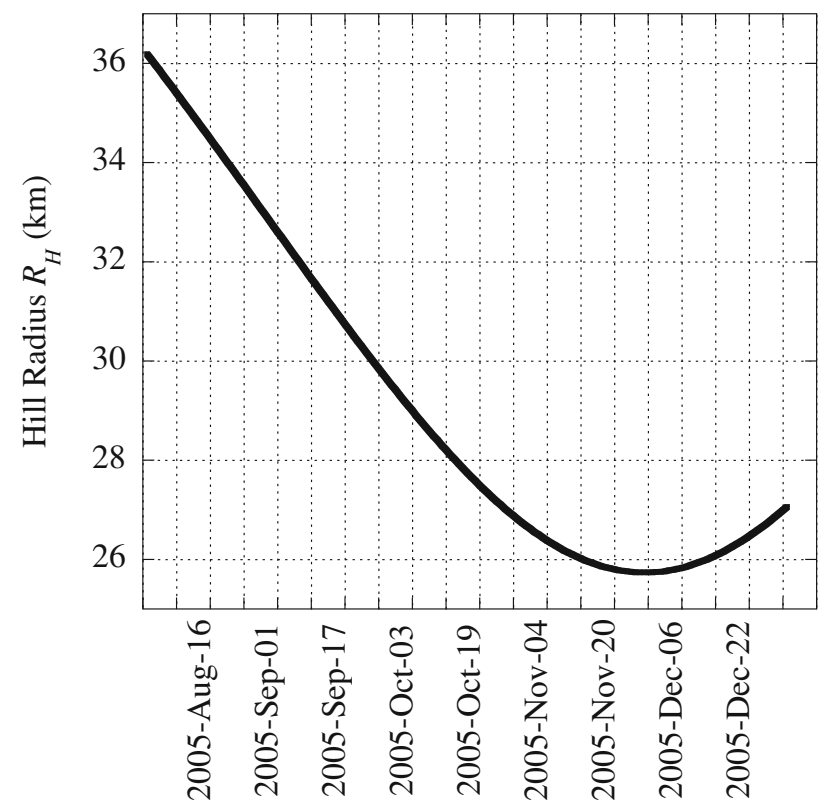

Date (UT)

Fig. 1. The variation of the Hill sphere $R_{H}$ of Itokawa that depends on its heliocentric distance $r$. Our observations were scheduled at the end of August and the beginning of September of 2005 during the spacecraft's approach phase to Itokawa.

$26 \mathrm{~km}$, and it implies that the stable orbital radius is less than or equal to $26 \mathrm{~km}$; however, we used the Hill radius of $33 \mathrm{~km}$, which was the value at our observation time, in order to detect debris that was recently trapped by Itokawa. Since AMICA has a $5.7 \times 5.7$ deg field-of-view (FOV) with a 1,024 × 1,024 pixel CCD (Saito et al., 2006), the image scale corresponds to a $100 \mathrm{~km} \times 100 \mathrm{~km}$ square image (at a range of $50 \mathrm{~km}$ from the center of Itokawa) with $100 \mathrm{~m}$ resolution at a distance of $1,000 \mathrm{~km}$ from Itokawa. This area fully covers the Hill sphere of Itokawa.

We next estimate the suitable interval time of the exposures. The circular orbital velocity $v$ of Itokawa's satellite is expressed as

$$
v=\sqrt{\frac{G\left(m+m^{\prime}\right)}{R}}
$$

where $m^{\prime}$ and $R$ are the mass (assumed $m^{\prime}=0$ here) and orbital radius of the satellite, respectively. An apparent orbital velocity from the spacecraft will be equal to or less than the orbital velocity by Eq. (2), because it depends on the relative position of the satellite to Itokawa; for example, the apparent velocity always looks the same when the orbital plane is face-on. As described previously, we originally estimated the distance of the spacecraft from Itokawa to be approximately $1,000 \mathrm{~km}$ (i.e. the pixel scale of AMICA was 
Table 2. Time is given for the observations on 2005/9/1 in UTC. $D_{I}, D_{E}$, and $D_{S}$ are the distances between the spacecraft and Itokawa, Earth and Sun, respectively, and S-I-S/C, S-E-S/C, and S-S/C-E are the angles of Sun-Itokawa-Spacecraft, Sun-Earth-Spacecraft, and Sun-Spacecraft-Earth, respectively on 2005/9/1 18:00:00 UTC. The exposure time is $66.7 \mathrm{sec}$ and the wide-bandpass filter was used for all observations.

\begin{tabular}{cccccccc}
\hline Frame ID & $\begin{array}{c}\text { Time in UTC } \\
2005 / 9 / 1\end{array}$ & $\begin{array}{c}D_{I} \\
(\mathrm{~km})\end{array}$ & $\begin{array}{c}D_{E} \\
(\mathrm{~km})\end{array}$ & $\begin{array}{c}D_{S} \\
(\mathrm{~km})\end{array}$ & $\begin{array}{c}\text { S-I-S/C } \\
(\mathrm{deg})\end{array}$ & $\begin{array}{c}\text { S-E-S/C } \\
(\mathrm{deg})\end{array}$ & $\begin{array}{c}\text { S-S/C-E } \\
(\mathrm{deg})\end{array}$ \\
\hline ST1 & $14: 11: 25$ & & & & & \\
ST2 & $16: 11: 10$ & & & & & \\
ST3 & $18: 11: 09$ & $1,660.8$ & 32,832 & 18,011 & 7.949 & & \\
ST4 & $20: 11: 09$ & & & & & \\
\hline
\end{tabular}

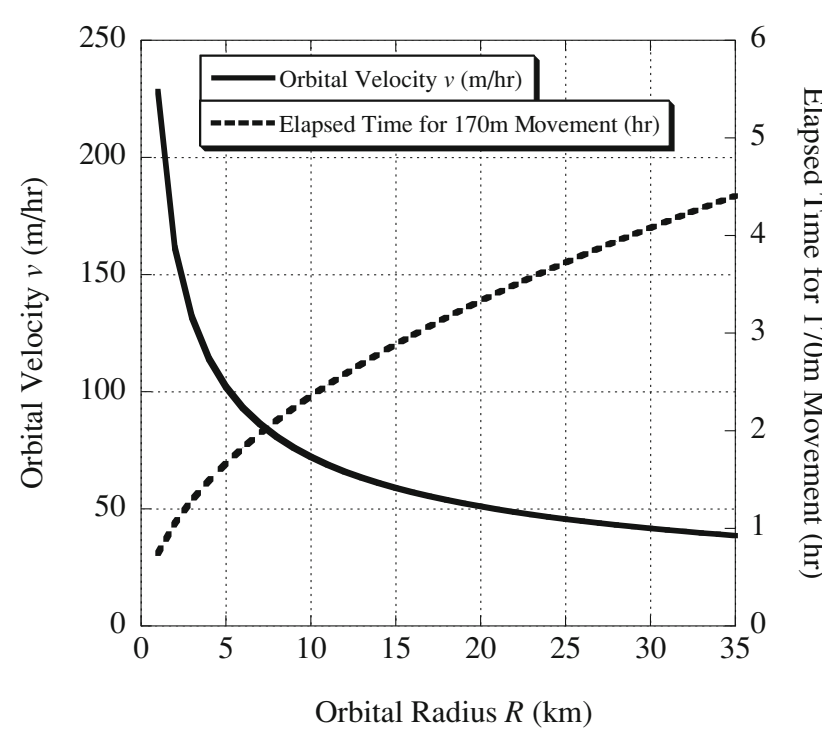

Fig. 2. (Left axis) The orbital velocity $v$ vs the orbital radius $R$ of a satellite of Itokawa, assuming the satellite has a circular orbit. (Right axis) The elapsed time for $170 \mathrm{~m}$ movement of the satellite, whose length corresponds to the pixel scale of AMICA at the observation. It is calculated by dividing $170 \mathrm{~m}$ by the orbital velocity $v$ shown on the left axis.

$100 \mathrm{~m}$ ), and the suitable exposure time was calculated by dividing $100 \mathrm{~m}$ by the orbital velocity $v$. The left axis of Fig. 2 indicates the relationship between the orbital radius $R$ and orbital velocity $v$. If the orbital radius is $33 \mathrm{~km}$, which is equal to the Itokawa's Hill sphere, it takes approximately $2.5 \mathrm{hr}$ to move $100 \mathrm{~m}$, which is the pixel scale. The ideal interval time is, therefore, more than $2.5 \mathrm{hr}$, but $2 \mathrm{hr}$ was finally chosen owing to the mission schedule.

The actual observation conditions are summarized in Table 2. AMICA has a wide-bandpass filter and seven narrow band filters (Saito et al., 2006); we used the wide-bandpass filter for collecting as many photons as possible. A longer exposure time is ideal for detecting smaller objects; however, one of three reaction wheels in the spacecraft's attitude control system had trouble and there was a possibility that the spacecraft would move during long exposures. Hence, the exposure time was set at $66.7 \mathrm{sec}$ by other test observations with AMICA. Since the actual distance between the spacecraft and Itokawa was approximately $1,700 \mathrm{~km}, \mathrm{AM}-$ ICA's FOV corresponded to $170 \mathrm{~km} \times 170 \mathrm{~km}$ (i.e. $85 \mathrm{~km}$ from Itokawa at the center of the image) and Itokawa's Hill sphere was completely inside the image at the new configuration.

\section{Results}

First and foremost, we must note that a huge solar flare occurred when the spacecraft took images of Itokawa on September 1, 2005. Energetic protons unfortunately left many spots on the four images (Table 2) as clearly seen in the median combined image in Fig. 3. Space Weather Highlight of 05-11 September 2005 (SWO PRF 1567)*1 reports "A large, complex sunspot group rotated into view on 07 September and was numbered as NOAA Region 808 (S09, L=232, class/area, Fkc/1430 on 09 September)." Assuming the time difference between our observation and the flare event resulted from the rotation of the Sun, it is probable that high-energy particles struck the spacecraft during imaging because the spacecraft faced toward the sunspots. The fact that many spots were observed on the optically dark area inside AMICA, which is insensitive to incident light, also supports our hypothesis. Since the noise by the particles randomly appears on the images, we believed it could be reduced during image processing.

Another concern from our observations was the rotation of the spacecraft due to trouble with the reaction wheel for the 2-hour interval of each exposure; AMICA is mounted toward the z-axis of the spacecraft and AMICA's field-ofview rotates clockwise at the center point on the image around the z-axis. Hence, Itokawa, stars, and possible satellites would appear at different positions on each image. Using a World Coordinate System (WCS) header of the FITS images, we arranged the ST2, ST3 and ST4 images relative to the ST1 image that was taken with the first exposure by rotating and shifting each of three images.

The final issue of note was the distance of the spacecraft from Itokawa during imaging; the actual distance of approximately $1,700 \mathrm{~km}$ (Table 2 ) was much longer than the expected value of $1,000 \mathrm{~km}$, which makes the detection of satellite movement difficult. The right axis of Fig. 2 shows the elapsed time for a satellite to move $170 \mathrm{~m}$, which is the exact pixel scale of our observation time. If the satellite has a $33 \mathrm{~km}$ orbital radius, which is the Hill sphere of Itokawa at that time, it needs $4 \mathrm{hr}$ to move $170 \mathrm{~m}$. Therefore, the $2 \mathrm{hr}$ interval observation period would be too short to find the motion of satellites. According to Fig. 2, we would not be able to detect the motion of a satellite whose orbital radius is larger than approximately $8 \mathrm{~km}$.

To achieve image quality, we applied the following reduction methods to the four original frames. Initially a dark frame was subtracted from each image, because the CCD

${ }^{* 1}$ Refer to Space Environment Center of NOAA at http://www.sec. noaa.gov/. 


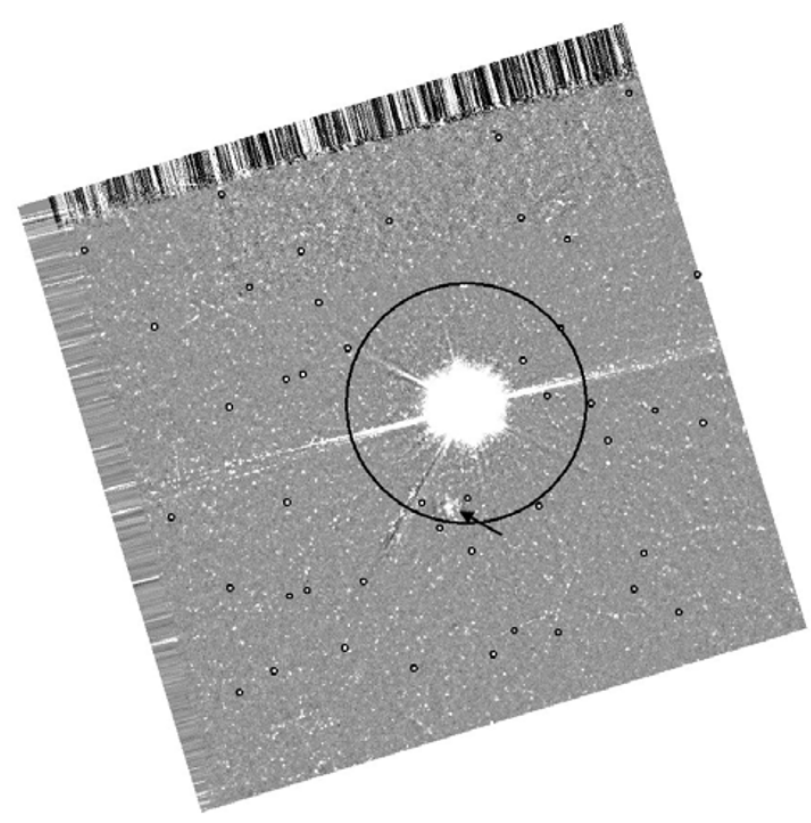

Fig. 3. The median combined image from the ST1, ST2, ST3, and ST4 images. North is up, and East is left. Itokawa is at the center of the bright glare. The large circle shows the Hill sphere of Itokawa at the observation time, corresponds to $33 \mathrm{~km}$ in radius. Many white spots were produced by the high energy protons from the large sun flare except for stars ( 9.5 mag) companioned by a small circle, whose positions are based on the Hipparcos Catalogue. The arrow indicates the ghost of Itokawa caused by scattering inside the AMICA optics.

chip of AMICA was not cooled sufficiently during imaging; its temperature was approximately -27 degrees Celsius. We made a median-combined image from the four images with an IRAF task, and then the median frame was subtracted from each of the four images. Next a flat field image, which was taken during a preflight test using an integrating sphere, was applied to the four median-subtracted images. After that, we carried out removal of cosmic rays using an IRAF command. From these tasks, four reduced images were obtained.

Subsequently, three reference stars in each image were selected to determine the rotation center and its angle (Table 3). In order to fit the three star positions on the ST2, ST3, and ST4 images to those on the ST1 image, we shifted and rotated the three images pixel by pixel. Finding the optimized rotation centers and angles for the ST2, ST3, and ST4 images, the three images were actually shifted and rotated based on the solutions with IRAF tasks. We finally combined the four images with the median filter of IRAF, and each star became a point source on the combined image.

Figure 3 displays the median combined image from the ST1, ST2, ST3, and ST4 images. Itokawa is buried in a bright glare that is 75 pixels in radius (i.e. $13 \mathrm{~km}$ in radius). The Hill sphere of Itokawa is marked with a large circle whose radius is $33 \mathrm{~km}$. Stars brighter than or equal to $9.5 \mathrm{mag}$ are plotted with small circles (based on the Hipparcos Catalogue) because the limiting magnitude of 9.5 is estimated by implanting artificial objects into the four original images and comparing the flux of them with that of cataloged stars. Visibly present is a bright mark near the south-
Table 3. Name is the Hipparcos Catalogue identifier. RA and Dec are the right ascension and declination (J2000.0), respectively, and V Mag is the magnitude of V-band based on the catalogue.

\begin{tabular}{cccc}
\hline Name & RA & Dec & V Mag \\
\hline HIP50333 & $10: 16: 40.75$ & $+13: 43: 42.1$ & 5.42 \\
HIP49637 & $10: 07: 54.32$ & $+09: 59: 51.6$ & 4.39 \\
HIP51213 & $10: 27: 38.99$ & $+09: 45: 44.7$ & 6.01 \\
\hline
\end{tabular}

ern edge of the Hill sphere. In some images of Itokawa, we found a ghost of Itokawa appeared symmetrically with respect to the CCD center. Accordingly, this bright mark is a ghost of Itokawa caused by scattering inside the optics of AMICA.

On the combined image many spots produced by the high energy particles from the large solar flare were observed. Since they could not be removed by image processing, we managed to distinguish the spots from stars by comparing the coordinate of each spot to that of stars listed in the Hipparcos Catalogue. The star positions were the same among the four images, while the spots were random positions on each image, and, as such, it was easy to distinguish between them.

Because the distance of the spacecraft from Itokawa was $1,700 \mathrm{~km}$ during imaging, the orbital radius of the satellites, detectable from their motions, must be less than $8 \mathrm{~km}$, and completely inside the bright glare. Movement could not be found among the images, and if a satellite were present it would appear as a star, with the same fixed position on each image. In as much as we could not find a moving object nor one in a fixed position that was not listed in the Hipparcos Catalogue, we concluded that a satellite was not present. Other Hayabusa observations as well as groundbased studies have also suggested an absence of Itokawa satellites.

\section{Discussion}

One of our study objectives was to estimate the size limit of detectable satellites for Itokawa. The diameter of a satellite, $D \mathrm{~km}$, is derived from the absolute magnitude $H_{0}$ and the geometric albedo $p_{v}$ (Bowell et al., 1989) with the following equation:

$$
\log _{10} D=0.5\left(6.259-0.4 H_{0}-2.5 \log _{10} p_{v}\right) .
$$

Lederer et al. (2005) estimated Itokawa's geometric albedo as $0.53 \pm 0.04$ in the V-band, which is much larger than the average albedo (0.22) for S-type asteroids. On the other hand, it is found that there is a large variation of $>30 \%$ in the V-band albedo as reported by the AMICA observations near Itokawa (Saito et al., 2006). Here we assume the satellite albedo $p_{v}$ to be the average value of 0.22 , although the satellite's albedo need not be similar to the average of S-type asteroids. The absolute magnitude $H_{0}$ of the satellite is given by (Jedicke et al., 2002; Bowell et al., 1989)

$$
H_{0}=V-5 \log _{10}\left(d r^{\prime}\right)+P(\alpha),
$$

where $V$ is the apparent magnitude of the satellite, $d$ in AU is the distance between the satellite and spacecraft, $r^{\prime}$ in AU is the heliocentric distance of the satellite, and $P(\alpha)$ is a 
scattering phase function at the phase angle of $\alpha$, which is "S-I-S/C" in Table 2. The distance $d$ is almost equal to $D_{I}$ in Table 2, and $r^{\prime}$ can be equal to the Itokawa's heliocentric distance $r$ at the observed time. Since the phase angle $\alpha$ is approximately $8 \mathrm{deg}$ from Table $2, P(\alpha)$ has a value small enough to neglect.

We now have the relationship between the diameter and magnitude of the satellite by Eqs. (3) and (4). Substituting these values (e.g. $V$ is assumed to be the limiting magnitude of 9.5 magnitude) into Eqs. (3) and (4), the satellite detection limit $D$ of $1 \mathrm{~m}$ in diameter is obtained. This indicates that our observation has ten times higher size-detectability than the radar observation by Ostro et al. (2004), whose resolution is a few decameters as noted in the introduction.

Saito et al. (2006) estimated the cumulative size distribution of boulders on Itokawa's surface with the AMICA observations and the slope of the cumulative size distribution is shallower than other small bodies in the solar system. The observations revealed more than 500 boulders, whose diameters are larger than $\sim 5 \mathrm{~m}$, spread over most of Itokawa's surface. The absence of orbiting boulders around Itokawa implies the existence of some removal mechanism from Itokawa's gravity such as a small escape velocity. The escape velocity $v_{\text {esc }}$ of orbiting objects from Itokawa is written as

$$
v_{\mathrm{esc}}=\sqrt{2} v=\sqrt{\frac{2 G\left(m+m^{\prime}\right)}{R}},
$$

where $G$ is the gravitational constant, and we obtain the $v_{\text {esc }}$ of several $\sim 1 \mathrm{~cm} / \mathrm{sec}$ from Fig. 2 . These values can be easily reached by physical phenomena such as collision, ejection, or fission. The shape of Itokawa observed by the Hayabusa spacecraft, in fact, suggests that Itokawa has experienced many large impacts (Fujiwara et al., 2006). These circumstances are consistent with the absence of a detectable satellite around Itokawa, confirmed by direct inspection by Hayabusa as well as by ground-based observatories.

\section{Summary}

On September 1, 2005 UTC, we conducted a search for satellites of asteroid Itokawa by imaging observations using AMICA on board the Hayabusa spacecraft. Four images, each of 2-hour intervals, were obtained over an 8-hour period. We found that the Hill sphere of Itokawa varied, and was estimated to be $33 \mathrm{~km}$ during imaging. The field-ofview of AMICA was approximately $170 \mathrm{~km}$ by $170 \mathrm{~km}$ and could fully cover the expected area of satellites; however, the region where a satellite's motion would be observable is only within $8 \mathrm{~km}$ of Itokawa, which is inside Itokawa's glare and we cannot see the movement of Itokawa satellites between the four images. Furthermore, it is unfortunate that a large solar flare occurred at the time of imaging, and the images contained many proton spots that could hardly be removed by image processing. After reducing the four im- ages, each was shifted and rotated in response to trouble with the spacecraft's reaction wheel, and the four images were then combined into a single image. Comparing the spots from the flare and stars listed in the Hipparcos Catalogue, we searched for Itokawa satellites on each image. No evidence for a satellite was found. The detection limit of 1 meter in diameter was derived from the limiting magnitude of our images. The low escape velocity and collision history of Itokawa may be responsible for small objects escaping the vicinity of Itokawa resulting in the absence of satellites. Our findings support this scenario.

Acknowledgments. We wish to thank the member of the Hayabusa operation team for providing the opportunity to carry out the satellite survey observation in a critical mission phase. We would especially like to acknowledge M. Nagano, T. Nakamura, T. Kubota and T. Hashimoto for their efforts in developing the AMICA. We would also like to thank Takashi Kominato of NEC Aerospace Systems, Ltd. for the navigation data of the Hayabusa spacecraft. The image processing was conducted by the computer system at Subaru Telescope, National Astronomical Observatory of Japan.

\section{References}

Belton, M. J. S. et al., The Discovery and Orbit of 1993 (243)1 Dactyl, Icarus, 120, 185-199, 1996.

Bowell, E. et al., Application of Photometric Models to Asteroids, in Asteroids II, edited by Binzel, R. P., T. Gehrels, and M. S. Matthews, 993 pp, Univ. of Arizona Press, Tucson, 1989.

Britt, D. T., D. Yeomans, K. Housen, and G. Consolmagno, Asteroids III, edited by Bottke Jr., W. F., A. Cellino, P. Paolicchi, and R. P. Binzel, 785 pp, Univ. of Arizona Press, Tucson, 2002.

Chapman, C. R. et al., Discovery and physical properties of Dactyl, a satellite of asteroid 243 Ida, Nature, 374, 783-785, 1995.

Fujiwara, A. et al., The Rubble-Pile Asteroid Itokawa as Observed by Hayabusa, Science, 312, 1330-1334, 2006.

Hamilton, D. P. and J. A. Burns, Orbital Stability Zones about Asteroids, Icarus, 92, 118-131, 1991.

Jedicke, R., J. Larsen, and T. Spahr, Asteroids III, edited by Bottke Jr., W. F., A. Cellino, P. Paolicchi, and R. P. Binzel, 785 pp, Univ. of Arizona Press, Tucson, 2002.

Lederer, S. M. et al., Physical characteristics of Hayabusa target Asteroid 25143 Itokawa, Icarus, 173, 153-165, 2005.

Melosh, H. J. and J. A. Stansberry, Doublet Craters and the Tidal Disruption of Binary Asteroids, Icarus, 94, 171-179, 1991.

Merline, W. J., S. J. Weidenschilling, D. D. Durda, J.-L. Margot, P. Pravec, and A. D. Storrs, Asteroids III, edited by Bottke Jr., W. F., A. Cellino, P. Paolicchi, and R. P. Binzel, 785 pp, Univ. of Arizona Press, Tucson, 2002.

Ostro, S. J. et al., Radar observations of asteroid 25143 Itokawa (1998 SF36), Meteoritics \& Planetary Sci., 39, 407-424, 2004.

Pravec, P. et al., Photometric survey of binary near Earth asteroids, Icarus, 181, 63-93, 2006.

Saito, J. et al., Detailed Images of Asteroid 25143 Itokawa from Hayabusa, Science, 312, 1341-1344, 2006.

Scheeres, D. J. et al., The Dynamical Environment About Asteroid 25143 Itokawa: Target of the Hayabusa Mission, AIAA/AAS Astrodynamics Specialist Conf. and Exhibit, AIAA 2004-4864, 1-12, 2004.

Williams, G. V., 1998 SF36, Minor Planet Electronic Circular, MPEC 1998-S45, 1998.

T. Fuse (e-mail: tetsu@subaru.naoj.org), F. Yoshida, D. Tholen, M. Ishiguro, and J. Saito 